ARTIGO DE REVISÃO

\title{
Obesidade infantil: revisão de literatura
}

\section{Child obesity: literature review}

\author{
Sofia Goersch Andrade Aragão ${ }^{1}$. \\ 1 Médica pediatra formada pelo Hospital Universitário Walter Cantídio (HUWC), Universidade Federal do Ceará (UFC), Fortaleza, \\ Ceará, Brasil.
}

\section{RESUMO}

A obesidade é um distúrbio crônico que resulta do aumento de gordura proveniente de um desequilíbrio no balanço energético. Vem acometendo cada vez mais crianças e adolescentes e está associada com graves consequências cardiovasculares e psicossociais. Com o objetivo de ampliar conhecimentos que favoreçam a prevenção e diagnóstico precoce de seus fatores de risco foram analisados estudos sobre obesidade infantil visando à promoção da saúde dessa faixa etária. A obesidade infantil se desenvolve, na maioria das vezes, da predisposição genética, associada a fatores comportamentais e ambientais. Verifica-se a grande importância da participação familiar, da prática de atividade física rotineira e do consumo de alimentos saudáveis e nutritivos desde o início da vida no combate à obesidade. A relevância deste agravo e suas consequências reforçam a necessidade de conscientização da prevenção de seus fatores de risco por meio de ações efetivas na primeira infância para inibir as tendências de crescimento da obesidade nesta faixa etária e provavelmente na fase adulta, dentre as quais se destacam as ações de puericultura, a avaliação do crescimento linear, assim como orientações regulares sobre controle e combate da obesidade aos pais e cuidadores, por serem estes os responsáveis pelo estado nutricional e comportamento alimentar infantil.

Palavras-chave: Obesidade. Peso corporal. Pediatria.

\section{ABSTRACT}

Obesity is a chronic disorder resulted of fat increasing from a disorder in the energy balance. It is increasingly affecting children and adolescents and it is associated with serious cardiovascular and psychosocial consequences. In order to expand the knowledge that promote the prevention and early diagnosis of their risk factors were analyzed studies on childhood obesity aiming health promotion in this age group. Childhood obesity comes from, in most cases, genetic predisposition, associated with behavioral and environmental factors. There is great relevance in family participation, physical activities and healthy and nutritious foods consumption since the beginning of life in the combat against obesity. The relevance of this disease and its consequences reinforce the necessity of risk factors prevention awareness through effective action in early childhood, to inhibit the growth of obesity in this age group and probably in adulthood, among which stand out childcare and its actions, linear growth evaluation, as well as parents and caregivers regular guidance on controling and combating obesity, since they are responsible for nutrition and the infant feeding behavior.

Keywords: Obesity. Body weight. Pediatrics.

Autor correspondente: Sofia Goersch Andrade Aragão, Rua Monsenhor Bruno, 2630, apartamento 401, Joaquim Távora, Fortaleza, Ceará. CEP: 60115-191.Telefone: +55 85 98881-2755. E-mail: su_goersch@hotmail.com

Conflito de interesses: Não há qualquer conflito de interesses por parte de qualquer um dos autores.

Recebido em: 03 Nov 2016; Revisado em: 01 Abr 2017; Aceito em: 01 Abr 2017. 


\section{INTRODUÇÃO}

A obesidade acomete a população adulta a bastante tempo, com diversas comorbidades associadas. Este agravo e suas graves consequências, até então mais prevalentes em adultos, hoje acomete também a faixa etária mais jovem. ${ }^{1,2} \mathrm{O}$ número de crianças e adolescentes em situação de sobrepeso e obesidade infantil vem crescendo nos últimos tempos, tornando-se um grave problema de saúde pública e interferindo na economia mundial. ${ }^{3}$ Na América Latina já são cerca de $20-25 \%$ da população total de crianças e adolescentes enfrentando esta condição. ${ }^{4}$

O Brasil não está longe desta realidade. Estudos mostram aumento na prevalência de obesidade e sobrepeso na população brasileira, com tendência de crescimento. ${ }^{5} \mathrm{~A}$ obesidade infantil já acomete em torno de $6,5 \%$ das crianças menores de 2 anos, ${ }^{6}$ $7,7 \%$ na faixa etária de 2 a 5 anos, $28,6 \%$ entre 5 e 9 anos e de $9,9 \%$ entre os adolescentes. ${ }^{7}$

Características antropométricas da população pediátrica podem ser consideradas como indicador de saúde, pois sofrem influência de fatores ambientais e comportamentais, que são mais importantes do que os fatores genéticos para expressão do potencial de crescimento durante os primeiros anos de vida. ${ }^{6}$

A obesidade na infância acarreta severas e variadas consequências para a saúde, podendo interferir na pressão arterial, no perfil lipídico, na resistência à insulina, na alteração do metabolismo da glicose, entre outros. ${ }^{3}$ A prevenção da obesidade e consequentemente de suas complicações podem interferir de sobremaneira na qualidade de vida dessas crianças. $^{8,9}$

A relevância deste agravo e suas graves consequências reforçam a necessidade de conscientização da prevenção e combate da obesidade em crianças e adolescentes. Este estudo apresenta ainda, dados que possibilitam reflexões para possíveis intervenções em saúde e melhorias em programas de puericultura para que se possa fazer o seguimento e tratamento dessas crianças.

\section{MATERIAL E MÉTODOS}

Foi realizada uma revisão da literatura através das bases de dados Scientific Eletronic Library Online (Scielo-Brasil), Pubmed, publicações do Ministério da Saúde e Diretrizes sobre o assunto. As palavras-chaves utilizadas para as buscas foram: "obesidade infantil", "obesidade em pediatria" e "alimentação infantil".

Os artigos foram escolhidos adotando-se como critério de inclusão aqueles disponíveis online, nas línguas portuguesa ou inglesa, publicados de 2000 até 2015 e que abordassem o tema obesidade infantil, sendo excluídos os que não entravam nestas categorias.

Foram selecionados 9 artigos, publicações do Ministério da Saúde do Brasil e da Sociedade Brasileira de Pediatria e a diretriz brasileira sobre obesidade para análise e utilização dos dados apresentados.

\section{RESULTADOS E DISCUSSÃO}

\section{Obesidade}

A obesidade é um distúrbio crônico que resulta do aumento de gordura proveniente de um desequilíbrio no balanço energético. ${ }^{5}$ Sua etiologia envolve fatores ambientais, genéticos e comportamentais. ${ }^{10}$ Pode estar associada a síndromes genéticas ou alterações endocrinometabólicas, sendo classificada como causa secundária, ou ser de causa primária, conhecida como obesidade exógena. ${ }^{5}$

\section{Fatores de risco}

Existem muitos estudos sobre as causas e os fatores predisponentes para sobrepeso e obesidade. Dados da literatura apontam que, quando um dos pais são obesos, as chances de a criança ser obesa duplica e triplica quando esta condição atinge os dois. ${ }^{11} \mathrm{~A}$ rápida recuperação do ganho de peso nos primeiros dois anos de vida também vem mostrando relação com obesidade. ${ }^{12}$ Essa relação também pode ser encontrada com o peso do nascimento, ou seja, bebês pequenos para idade gestacional (PIG) ou grandes para idade gestacional (GIG), além de um risco aumentado para doenças cardiovasculares. ${ }^{5}$

O aleitamento materno vem sendo apontado como fator protetor, ${ }^{5}$ deixando sob maior risco crianças que sofrem desmame precoce e acabam sendo expostas a alimentos inadequados e uso de fórmulas lácteas diluídas erroneamente. ${ }^{13}$

Os fatores genéticos podem interferir no desenvolvimento da obesidade, no entanto os principais determinantes são os fatores comportamentais e ambientais, como o sedentarismo, maior tempo em telas, balanço energético positivo e aumento do consumo de alimentos com alto valor glicêmico e calórico e pouco valor nutricional em substituição a alimentos saudáveis. As relações e os hábitos familiares influenciam diretamente a situação nutricional e a atitude da criança diante do alimento, sendo responsável pela formação do comportamento alimentar. ${ }^{2}$ Estudos demostram que os alimentos ofertados às crianças influenciam no desenvolvimento pondero-estatural, interferindo na formação do tecido adiposo. ${ }^{12}$

\section{Morbidade}

A alta prevalência da obesidade vem contribuindo para o aparecimento das complicações associadas em idades cada vez mais precoces. ${ }^{10}$

A associação deste agravo com doenças crônicas como a hipertensão arterial, diabetes tipo 2, dislipidemia, intolerância à glicose, resistência à insulina, puberdade precoce e distúrbios do sono está cada vez mais frequente segundo estes estudos. ${ }^{14}$ Sabe-se que a doença aterosclerótica tem início já na infância e adolescência, assim como demonstram relação entre gordura abdominal e alterações metabólicas. ${ }^{5}$ As alterações psicossociais também merecem atenção e costumam acometer mais os adolescentes. ${ }^{2}$ 
O desenvolvimento dessas comorbidades tem grande impacto na qualidade de vida e na morbimortalidade das crianças e adolescentes. $^{14}$

\section{Avaliação}

$\mathrm{Na}$ avaliação do peso de crianças pode-se usar o índice de massa corpórea (IMC) como forma de aferir a gordura corporal e o perfil nutricional. É calculado com base no peso e altura, através da fórmula: IMC = peso $(\mathrm{kg}) /$ estatura $(\mathrm{m})^{2}$; sendo classificado como sobrepeso valores entre o percentil 85 e 95, e como excesso de peso, acima do percentil 95, sendo obesidade grave aqueles que estão acima do percentil 97 para as curvas de idade cronológica. O IMC também pode ser expresso por meio de escores z ou desvios-padrão, considerado como obesidade os valores situados acima do +2 desvios-padrão e como obesidade grave valores acima do +3 desvios-padrão. ${ }^{10}$

Como toda consulta de puericultura, a avaliação do crescimento linear é fundamental e pode apresentar informações sobre o estado nutricional da criança. ${ }^{8}$ Medidas como prega cutânea tricipital (PCT) e circunferência do braço (CB) também podem ser úteis nesta avaliação. Outra medida utilizada e de extrema importância para crianças é a circunferência abdominal (CA) que avalia de forma indireta a gordura visceral, diagnosticando obesidade central. ${ }^{10}$ Ainda não existem valores de referência específicos para crianças e adolescentes. Segundo as Diretrizes Brasileiras de Obesidade, medidas de CA acima de 71 conferem um maior risco a doenças cardiovasculares. ${ }^{5}$

\section{Tratamento}

São poucos os dados sobre o tratamento e o melhor momento de intervenção quando se trata de obesidade na faixa etária pediátrica. ${ }^{11}$ A literatura é escassa e pouco

\section{REFERÊNCIAS}

1. Ministério da Saúde (Brasil). Vigitel Brasil 2009: vigilância de fatores de risco e proteção para doenças crônicas por inquérito telefônico [Internet]. Brasília: Ministério da Saúde; 2010 [acesso em: 17 agosto 2016]. Disponível em: http://189.28.128.100/dab/docs/ publicacoes/geral/publicacao_vigitel_2009.pdf

2. Gomes MJ, Nascimento EG. The multiple facets of overweight in child: a systematic review. Revista de Atenção à Saúde. 2015;13(45):70-9.

3. Onis M. Preventing childhood overweight and obesity. J Pediatr (Rio J). 2015;91(2):105-7.

4. Rivera JA, Cossío TG, Pedraza LS, Aburto TC, Sánchez TG, Martorell R. Childhood and adolescent overweight and obesity in Latin America: a systematic review. Lancet Diabetes Endocrinol. 2014;2(4):321-32.

5. Associação Brasileira para o Estudo da Obesidade e da Síndrome Metabólica (ABESO). Diretrizes brasileiras de obesidade 2009/2010 [Internet]. 3. ed. Itapevi (SP): AC Farmacêutica; 2009 [acesso em: 20 agosto 2016]. Disponível em: http://www.abeso.org.br/pdf/ diretrizes_brasileiras_obesidade_2009_2010_1.pdf conclusiva. O que se têm são orientações sobre controle do ganho de peso associado a mudanças do estilo de vida e monitorização das comorbidades encontradas. ${ }^{5}$

A participação da família nesse processo é de fundamental importância e a mudança deve ocorrer em todos os membros. A busca por uma alimentação mais saudável, com alimentos de menor índice glicêmico e calórico, maior ingestão de frutas e verduras, menor ingestão de industrializados e um aumento do gasto energético com atividades físicas devem fazer parte da rotina de todos em casa. ${ }^{5}$ Estudos referem a atividade física programada como importante na redução da gordura corporal. ${ }^{3}$ No Brasil já está liberado o uso de fármacos como a sibutramina para maiores de 16 anos e nos Estados Unidos o orlistat está sendo usado para adolescentes. O tratamento medicamentoso e a cirurgia bariátrica ainda não são embasados por estudos para essa faixa etária, devendo-se pesar os riscos e benefícios quando esta for a única alternativa. ${ }^{5}$

\section{CONCLUSÃO}

A partir desses dados, pode-se perceber que a obesidade é um distúrbio médico-social bastante complexo e multifatorial, podendo causar diversos prejuízos na infância. Pode-se dizer que se tornou uma epidemia, sendo uma questão de saúde pública mundial.

É necessária implantação de medidas para o combate e prevenção deste agravo nutricional. As ações de puericultura, a prática de atividades físicas e hábitos alimentares saudáveis, assim como orientações aos pais e cuidadores são fundamentais para o combate e controle da obesidade. Crianças com excesso de peso têm risco acrescido de se tornarem adultos obesos e, as consequências advindas dessa patologia podem onerar muito os custos em saúde.

6.Coccetti M, Taddei JA, Konstantyner T, Konstantyner TC, Barros AA Filho. Prevalence and factors associated with overweight among Brazilian children younger than 2 years. J Pediatr (Rio J). 2012;88(6):503-8.

7. Ministério da Saúde (Brasil). Pesquisa de orçamentos familiares 2008-2009: antropometria e estado nutricional de crianças, adolescentes e adultos no Brasil. Brasília; 2010.

8. Ministério da Saúde (Brasil). Obesidade. Brasília; 2006. (Cadernos de atenção básica; n. 12); (Série A, Normas e manuais técnicos).

9. Gobato AO, Vasques AC, Zambon MP, Barros AA Filho, Hessel G. Metabolic syndrome and insulin resistance in obese adolescents. Rev Paul Pediatr. 2014;32(1):55-62.

10. Sociedade Brasileira de Pediatria. Departamento de Nutrologia. Obesidade na infância e adolescência: manual de Orientação. 2. ed. São Paulo: SBP; 2012.

11. Dubois L, Girard M. Early determinants of overweight at 4.5 years in population-based longitudinal study. Int J Obes (Lond). 2006;30(4):610-7. 
12. Matos SM, Jesus SR, Saldiva SR, Prado MS, D’Innocenzo S, Assis AM, et al. Weight gain rate in early childhood and overweight in children 5-11 years old in Salvador, Bahia State, Brazil. Cad Saúde Pública. 2011;27(4):714-22.

13. Fisberg M, Baur L, Chen W, Hoppin A, Koletzko B, Lau D, et al. Obesity in children and adolescents: Working Group Report of the
Second World Congress of Pediatric Gastroenterology, Hepatology, Nutrition. J Pediatr Gastroenterol Nutr. 2004;(39 Supl 2): S678-87.

14. Villa JK, Silva AR, Santos TS, Ribeiro AQ, Sant'Ana LF. Risco de síndrome metabólica em crianças: uso de um escore único. Rev Paul Pediatr. 2015;33(2):187-93.

\section{Como citar:}

Aragão SG. Obesidade infantil: revisão de literatura. Rev Med UFC. 2017 set-dez;57(3):47-50. 